\title{
Lateral band formation and hybridization in molecular monolayers: NTCDA on $\operatorname{Ag}(110)$ and $\mathrm{Cu}(100)$
}

\author{
M. Wießner, ${ }^{1,2}$ J. Kübert, ${ }^{1}$ V. Feyer, ${ }^{3,4}$ P. Puschnig, ${ }^{5}$ A. Schöll,,${ }^{1,2, *}$ and F. Reinert ${ }^{1,2}$ \\ ${ }^{1}$ Universität Würzburg, Experimentelle Physik VII and Röntgen Research Center for Complex Material Systems, 97074 Würzburg, Germany \\ ${ }^{2}$ Karlsruher Institut für Technologie, Gemeinschaftslabor für Nanoanalytik, 76021 Karlsruhe, Germany \\ ${ }^{3}$ Peter Grünberg Institute (PGI-6) and Jülich Aachen Research Alliance, Fundamentals of Future Information Technology, \\ Research Center Jülich, 52425 Jülich, Germany \\ ${ }^{4}$ Sincrotrone Trieste, NanoESCA Beamline, Area Science Park, 34149 Basovizza, Trieste, Italy \\ ${ }^{5}$ Institut für Physik, Karl-Franzens-Universität Graz, 8010 Graz, Austria
}

(Received 24 July 2013; published 29 August 2013)

\begin{abstract}
The adsorption of aromatic molecules on metal surfaces leads to a complex reorganization of the molecular and metal wave functions. Various processes such as charge transfer, hybridization between molecular and metallic states, and the formation of dispersing bands within the interface have been demonstrated for organometallic interface systems. For the model molecule 1,4,5,8-naphthalenetetracarboxylic dianhydride (NTCDA), we compare highly ordered monolayers on $\mathrm{Ag}(110)$ and $\mathrm{Cu}(100)$, which allows us to identify changes of the interfacial electronic structure when altering the coupling strength with the substrate by means of angle-resolved photoelectron spectroscopy. The stronger coupling to the $\operatorname{Ag}(110)$ substrate goes along with a shorter photohole lifetime and a stronger hybridization of the NTCDA lowest unoccupied molecular orbital with metal states. Supported by ab initio calculations, we show that the observed band dispersion is greatly enhanced due to the interaction with $\operatorname{Ag}(110)$ while the laterally denser adsorption geometry of NTCDA on $\mathrm{Cu}(100)$ entails a larger intermolecular wave-function overlap, and the presence of the substrate results in no further bandwidth enhancement.
\end{abstract}

DOI: 10.1103/PhysRevB.88.075437

PACS number(s): 33.60.+q, 73.20.Hb, 68.43.Pq, 79.60.Dp

\section{INTRODUCTION}

Organic semiconductors are particular materials for active layers in optoelectronic devices. Low-cost production, effective light absorption and emission, as well as the tunability of the molecular building blocks make them interesting for various applications. ${ }^{1-4}$ However, in addition to the molecular properties, interfaces to metallic contacts are decisive for the device performance. Especially, the charge injection and extraction and the transport in the vicinity of the contact interface are crucial for the electronic properties of the resulting organometallic systems. In the case of large $\pi$-conjugated molecules, a multitude of publications report on interfaces to different metals. ${ }^{5-11}$ Some parameters such as charge injection barriers or ionization potentials can be straightforwardly determined from photoelectron spectroscopy (PES). ${ }^{12-14}$ Moreover, the bonding at metal-organic interfaces also leads to the generation of novel states, ${ }^{5,15-19}$ which are due to the hybridization of metal and molecular wave functions. These can be identified and characterized by angle-resolved PES (ARPES). ${ }^{20,21}$

Here we study the well-known model system 1,4,5,8-naphthalenetetracarboxylic dianhydride (NTCDA) on $\mathrm{Ag}(110)$ and $\mathrm{Cu}(100)$ single-crystal surfaces by means of ARPES. Supported by calculations within the framework of density-functional theory (DFT), we show that the hybridization at metal-organic interfaces can be the source of very particular phenomena in the electronic structure. In the case of NTCDA/Ag(110) a strong interfacial bonding occurs, which involves a marked hybridization of the NTCDA lowest unoccupied molecular orbital (LUMO) with metal states and a substrate-mediated delocalization of the resulting hybrid state. The latter manifests itself in an increased lateral band dispersion of the $\mathrm{LUMO}^{21}$ and a shorter lifetime as evidenced by an enhanced peak width. Considering experience with similar systems, ${ }^{7,22}$ the bonding in the case of $\mathrm{NTCDA} / \mathrm{Cu}(100)$ is expected to be even stronger. Interestingly, however, we find that the lateral band dispersion for NTCDA/Cu(100) is mainly a result of the direct intermolecular interaction due to the much denser packing of the molecules as compared to NTCDA/Ag(110). Thus, while the magnitudes of the observed band dispersions on the two substrates are quite similar, the underlying physics involved in hybridization and lateral band formation are quite different.

\section{EXPERIMENTAL AND COMPUTATIONAL DETAILS}

All experiments were performed in an ultrahigh vacuum system with a base pressure of $5 \times 10^{-10}$ mbar. The NTCDA films were prepared in an attached preparation chamber by organic molecular-beam deposition with a deposition rate of about 0.8 monolayer (ML)/min onto clean and wellordered $\mathrm{Ag}(110)$ and $\mathrm{Cu}(100)$ single-crystal surfaces ${ }^{23}$ at room temperature.

The ARPES measurements were obtained with a monochromatized UV lamp for $\mathrm{He} \mathrm{II}_{\alpha}$ radiation $(h v=$ $40.8 \mathrm{eV}$ ) and with radiation from the HiSOR synchrotron at beamline 9 A. Together with a hemispherical photoelectron analyzer (Scienta SES200, R4000) the overall energy resolution was on the order of about $\Delta E=10 \mathrm{meV}$. With this setup the angle-resolved data were recorded by rotating the sample around the manipulator axis as indicated by the arrows in Fig. 3. The $k_{\|}$-dependent photoemission intensity distributions have been obtained at the NanoESCA beamline at Elettra $^{24}$ using a FOCUS GmbH/Omicron NanoESCA electron spectrometer. ${ }^{25,26}$ The setup includes a nonmagnetic, 
(a)

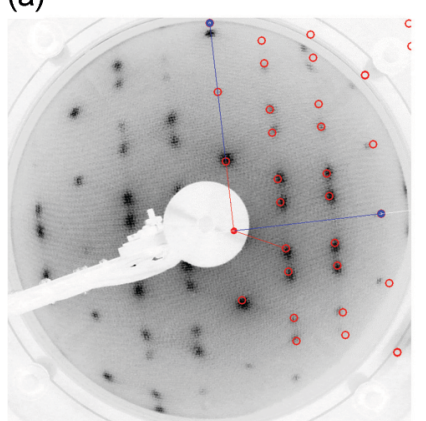

(b)

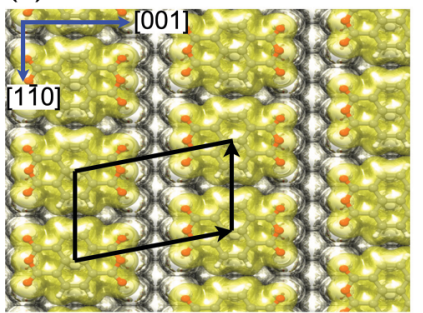

(c)

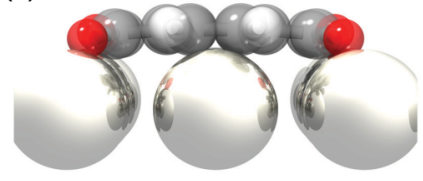

(d)

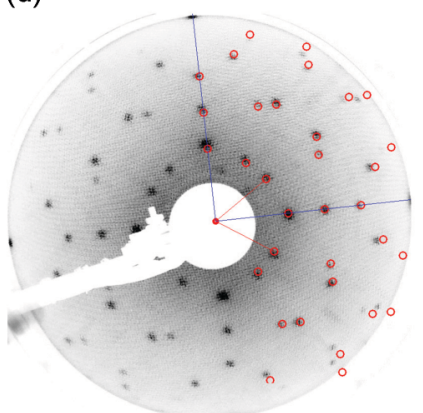

(e)

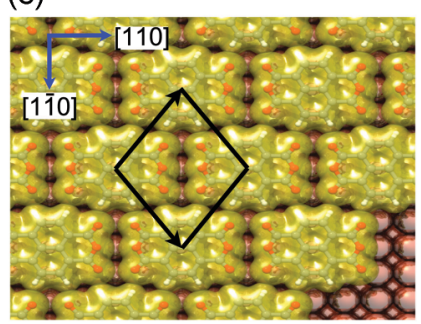

(f)

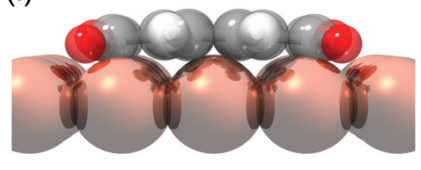

FIG. 1. (Color online) Low electron energy diffractograms of 1 ML NTCDA (a) on Ag(110) using $51 \mathrm{eV}$ and (d) on $\mathrm{Cu}(100)$ using $55 \mathrm{eV}$ of electron-beam energy. (b), (e) Corresponding lateral real space models. The unit cell of each structure is indicated by the black arrows, and the substrate high-symmetry directions are indicated by the blue arrows. (c), (f) Calculated adsorption geometry of the NTCDA molecule on the two substrates (c) $\mathrm{Ag}(110)$ and (f) $\mathrm{Cu}(100)$.

electrostatic photoelectron emission microscope and an aberration compensated double-pass hemispherical analyzer. This instrument is equipped with a transfer lens behind the immersion lens objective to map the angular distribution of the photoelectrons by imaging the focal plane. A detailed description of the measurement and evaluation process can be found in Ref. 27.

Figure 1 shows the low-energy electron-diffraction (LEED) diffractograms of 1 ML NTCDA on $\operatorname{Ag}(110)$ (a) and on $\mathrm{Cu}(100)$ (d). On both substrates it is well known that the molecules grow in highly ordered brickwall arrangements. For the $\operatorname{Ag}(110)$ substrate a $\left(\begin{array}{ll}3 & 0 \\ 1 & 3\end{array}\right)$ superstructure can be observed with one molecule and an area of $106.4 \AA^{2}$ per unit cell. ${ }^{28}$ The resulting real space model is sketched in Fig. 1(b). The brickwall arrangement found on $\mathrm{Cu}(100)$ is somewhat different. The LEED [Fig. 1(d)] features a superstructure given by $\left(\begin{array}{cc}2 & -3 \\ 2 & 3\end{array}\right)$ associated to a denser monolayer with one molecule and $76.2 \AA^{2}$ per unit cell. The respective real space model is given in Fig. 1(e). In contrast to NTCDA/Ag(110), two possible orientations of the molecular domains exist on $\mathrm{Cu}(100)$ due to the fourfold symmetry of the latter substrate. DFT calculations for NTCDA/Ag(100) and NTCDA/Cu(100) are carried out with the VASP code $^{29,30}$ using a generalized gradient approximation for exchange-correlation effects ${ }^{31}$ and

empirical van der Waals corrections according to Grimme. ${ }^{32}$ The projector augmented waves ${ }^{33}$ approach was used, allowing for a relatively low kinetic-energy cutoff of about $400 \mathrm{eV}$. The $\mathrm{Ag}(110) / \mathrm{Cu}(100)$ substrates are modeled by six layers of $\mathrm{Ag} / \mathrm{Cu}$, respectively, with an additional vacuum layer of about $16 \AA$. For NTCDA/Ag(110) and NTCDA/Cu(100), we use Monkhorst-Pack ${ }^{34} k$-point grids of $8 \times 6 \times 1$ and $8 \times 8 \times 1$, respectively, and a first-order Methfessel-Paxton smearing of $0.2 \mathrm{eV}^{35}$ To avoid spurious electrical fields, a dipole layer is inserted in the vacuum region of the asymmetric slab. ${ }^{36}$ For the evaluation of the band dispersion of molecule-derived bands, we analyze the $k$-resolved density of states projected onto the molecular orbital under investigation.

The ARPES momentum space patterns of the highest occupied molecular orbital (HOMO) and LUMO have been calculated for a free-standing NTCDA molecule by assuming a plane-wave final state following a procedure described previously. ${ }^{37}$ For these computations the planar geometry of an isolated NTCDA molecule has been assumed. We note that the geometrical deformations of the molecule caused by the adsorption on the metallic surface ${ }^{38}$ induce only minor changes in the HOMO and LUMO $k$-space patterns as has been shown for the similar PTCDA/Ag(110) system. ${ }^{39}$

\section{RESULTS AND DISCUSSIONS}

\section{A. Photoemission line shape}

Figure 2 shows a comparison of PES spectra recorded for NTCDA/Ag(110) (top) and NTCDA/Cu(100) (bottom) at $k_{[001]}=0, k_{[1 \overline{1} 0]}=1.5 \AA^{-1}$ and at $k_{[110]}=0, k_{1 \overline{1} 0}=1.7 \AA^{-1}$, respectively. In case of NTCDA/Ag(110) two signals originating from the adsorbate molecules can clearly be identified. The signal at binding energy $E_{B}=2.4 \mathrm{eV}$ can be associated with the HOMO. The peak at $E_{B}=0.5 \mathrm{eV}$ only appears in PES spectra from the first monolayer and can be attributed to the LUMO, which is occupied by charge transfer from the substrate. ${ }^{5,15,20}$ This is unambiguously demonstrated by the $k_{\|}$-dependent photoelectron intensity distributions shown in Fig. 3. As demonstrated in literature and by the Fourier transforms of the NTCDA HOMO and LUMO shown in Fig. 3, the intensity patterns can be interpreted as the square of the Fourier transforms of the wave function in real space, ${ }^{37}$ which allows a straightforward identification of the respective molecular orbitals..$^{20,27,40,41}$ Additionally, Fig. 3 clarifies at which positions in $k$ space the PES spectra of Fig. 2 were recorded. The gray (white) point indicates the $k_{\|}$position of one of the intensity maxima of the LUMO for NTCDA on $\operatorname{Ag}(110)[\mathrm{Cu}(100)]$. The LUMO also has a binding-energy minimum at these positions in $k$ space (see Sec. III C for details). Note that the HOMO shows only weak PES intensity at this position due to the different orbital symmetry which is evident from Fig. 3.

While the HOMO cannot straightforwardly be identified for NTCDA/Cu(100) since it is superimposed by the very intense onset of the Cu- $3 d$ states starting at about $E_{B}=2.0 \mathrm{eV}$, the occupied LUMO appears at $E_{B}=0.66 \mathrm{eV}$, i.e., at a slightly larger binding energy compared to the monolayer on $\mathrm{Ag}(110)$.

Additional information can be derived from an analysis of the line shape of the molecular signals. It is well known that for 


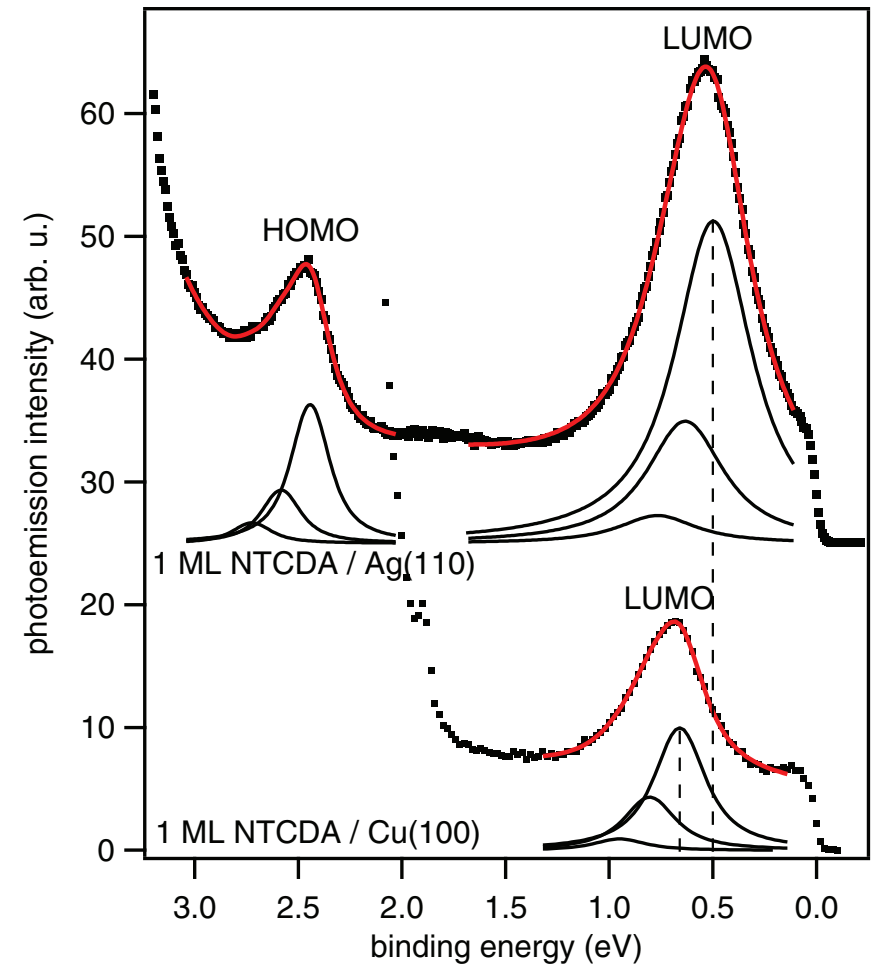

FIG. 2. (Color online) PES spectra for NTCDA/Ag(110) recorded at $h v=36 \mathrm{eV}, k_{1 \overline{1} 0}=1.5 \AA^{-1}, T=100 \mathrm{~K}$ (top) and for $\mathrm{NTCDA} / \mathrm{Cu}(100)$ recorded with $\mathrm{He} \mathrm{II}_{\alpha}$ radiation, $k_{1 \overline{1} 0}=1.7 \AA^{-1}$, $T=60 \mathrm{~K}$ (bottom). Below the spectra the results of peak fit analyses of the respective signals using three vibronic states are displayed. The sum curves derived from the fits are plotted as red lines. See text for details.

organic solids the line shape of PES signals is determined by several mechanisms. ${ }^{43}$ For the two well-ordered monolayer systems NTCDA on $\operatorname{Ag}(110)$ and on $\mathrm{Cu}(100)$, however, we can certainly assume that the influences of inhomogeneities and defects are similar and can thus be neglected in a relative comparison. Line-shape differences can then be mainly assigned to two effects: the hole-vibration coupling ${ }^{44-47}$ and the lifetime of the photohole. ${ }^{45}$ While the former leads to a typical asymmetry of the PES signals from molecular states, the latter manifests itself in a Lorentzian contribution to the line shape. Several publications demonstrate the potential of a PES line-shape analysis based on a single-mode analysis to determine vibrational energies, hole-vibration coupling parameters, ${ }^{44-46}$ and hole lifetimes. ${ }^{48,49}$ The majority of that work has been done for very well-ordered molecular layers on weakly interacting substrates such as highly oriented pyrolytic graphite (HOPG).

We applied a similar analysis to our data. The PES signals were fitted by vibrational progressions of Voigt peaks. Constraints were utilized such that the peak-to-peak distances $E_{\text {vib }}$ and Lorentzian linewidths $\Gamma_{(L)}$ are identical within each progression. During the fit analysis the Gaussian contribution $\Delta_{(G)}$ to the line shape turned out to be much smaller than the $\Gamma_{(L)}$ contribution, rendering a quantification insignificant. $\Delta_{(G)}$ was thus fixed in the further analysis to a value of $91 \mathrm{meV}$, which was derived from PES experiments in the gas phase. ${ }^{42}$ Figure 2 shows the result of these fits on the HOMO and (a)

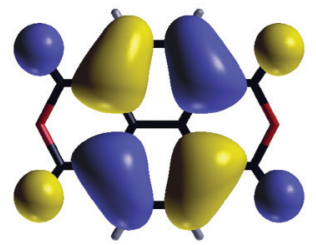

(b)

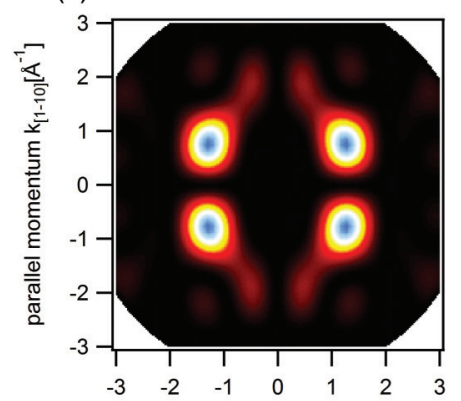

(c)

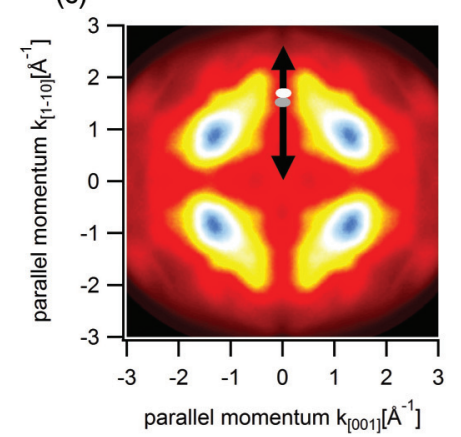

(d)

(e)
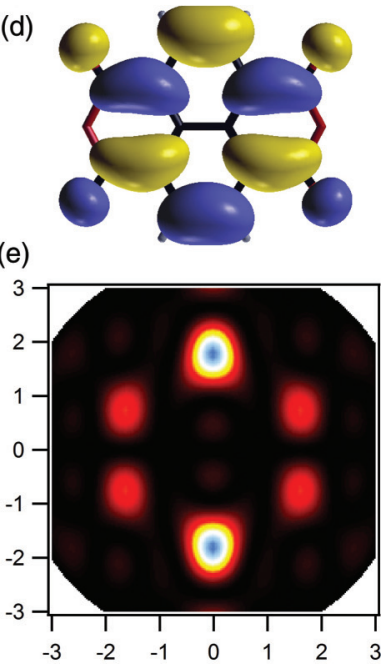

(f)

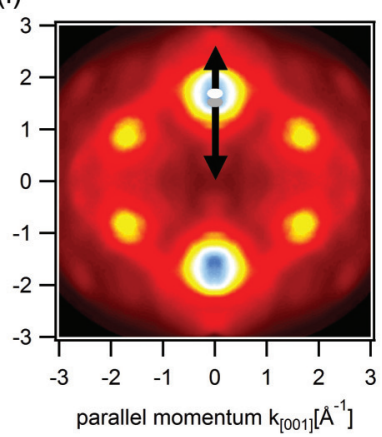

FIG. 3. (Color online) Calculated (a), (d) real space and (b), (e) Fourier space representation for a kinetic energy of $50 \mathrm{eV}$ of the HOMO (left) and LUMO (right) of an isolated NTCDA molecule. (c), (f) Respectively, the $k_{\|}$-dependent photoelectron intensity patterns recorded within a binding-energy window of $\Delta E=200 \mathrm{meV}$ for the (c) HOMO and (f) LUMO of NTCDA/Ag(110) $(h v=55 \mathrm{eV}$, $p$ polarization, room temperature). The black arrows illustrate the direction at which the ARPES momentum distributions of Fig. 4 were derived. The gray and white points indicate the $k_{\|}$positions of the LUMO binding-energy minima referred to in Fig. 2. See text for details.

LUMO signals plotted below the respective PES spectra. The fitting parameters are summarized in Table I. For all three signals three vibrational states can be resolved. The spacing $E_{\text {vib }}$ of the vibronic peaks is around $140 \mathrm{meV}$ in all three cases. In the gas phase the NTCDA HOMO shows a slightly larger peak separation of $153 \mathrm{meV}^{42} \mathrm{~A}$ similar behavior has been observed for other adsorbate systems such as pentacene, which shows $158 \mathrm{meV}$ on HOPG compared to $167 \mathrm{meV}$ in the gas phase. In the latter case this softening of the vibronic potential was related to the molecule-substrate electronic coupling ${ }^{49-52}$ and an explanation along this line may also be considered in our case. The relative intensities of the vibronic components, which are described by the Huang-Rhys factor $S$, is closer to the gas phase value for NTCDA adsorbed on $\mathrm{Cu}$ than on $\mathrm{Ag}$. The resulting reorganization energies $\lambda$ for NTCDA, derived by $\lambda=2 \cdot S h v,{ }^{45}$ are 107 and $108 \mathrm{meV}$ for the LUMO and HOMO on $\mathrm{Ag}(110)$, respectively. For the LUMO on $\mathrm{Cu}(100)$ a value of $126 \mathrm{meV}$ can be determined, while $\lambda=160 \mathrm{meV}$ for the HOMO in the gas phase. 
TABLE I. Fitting coefficients for the deconvolution of the NTCDA HOMO and LUMO signals. $E_{B}$ gives the binding energy of the lowest vibronic state, $E_{\text {vib }}$ gives the spacing within the vibronic progressions, $\Gamma_{(L)}$ gives the Lorentzian FWHM, and $\Delta_{(G)}$ gives the Gaussian FWHM contribution to the utilized Voigt profiles. The Huang-Rhys factor $S$ was determined from the intensity ratio of the respective $0-0$ and $0-1$ transitions. The gas phase parameters were extracted from Ref. 42.

\begin{tabular}{lccccc}
\hline \hline & $\begin{array}{c}E_{B} \\
(\mathrm{eV})\end{array}$ & $\begin{array}{c}E_{\mathrm{vib}} \\
(\mathrm{meV})\end{array}$ & $\begin{array}{c}\Gamma_{(L)} \\
(\mathrm{meV})\end{array}$ & $\begin{array}{c}\Delta_{(G)} \\
(\mathrm{meV})\end{array}$ & $S$ \\
\hline HOMO Ag(110) & 2.44 & $140 \pm 30$ & $190 \pm 50$ & 91 & 0.40 \\
LUMO Ag(110) & 0.50 & $135 \pm 15$ & $427 \pm 30$ & 91 & 0.41 \\
LUMO Cu(100) & 0.66 & $145 \pm 15$ & $270 \pm 30$ & 91 & 0.54 \\
HOMO gas ph. ${ }^{42}$ & - & $153 \pm 4$ & 37 & 91 & 0.51 \\
\hline \hline
\end{tabular}

An even larger difference between the different PES signals becomes apparent if the Lorentzian widths $\Gamma_{(L)}$ are compared. $\Gamma_{(L)}$ is much larger for the LUMO in the case of NTCDA/Ag(110) (427 meV) than in the case of NTCDA/Cu(100) $(270 \mathrm{meV})$ and also much larger than for the HOMO on $\operatorname{Ag}(110)(190 \mathrm{meV})$ and for the HOMO of the isolated molecule $\left(37 \mathrm{meV}^{42}\right)$. In the simplest approach, $\Gamma_{(L)}$ can be related to the lifetime of the photohole and an upper limit for the lifetime can be calculated using the uncertainty relation. ${ }^{45,53}$ Consequently, differences in $\Gamma_{(L)}$ can be related to differences in the coupling between the photohole and the substrate electrons. Note that the spectra were derived by integrating over a $k_{\|}$range of $\Delta k=0.1 \AA^{-1}$. The resulting influence of the intermolecular band dispersion can be estimated to be smaller than $50 \mathrm{meV}$ and very similar for both systems (see also Sec. III C). Moreover, the effect of electron-phonon coupling can be ruled out to be dominating in this case since it leads to a slight increase of the linewidth with increasing energetic distance from the Fermi energy. ${ }^{54} \mathrm{~A}$ possible explanation for the observed shortest lifetime for the LUMO in the case of NTCDA/Ag(110) can be given on the basis of the coupling between the molecular electronic system and the substrate. According to this argument, this coupling would be strongest in the case of NTCDA/Ag(110).

\section{B. Parallel momentum dependent intensity distribution}

As insightfully demonstrated on the example of the similar molecule 3,4,9,10-perylenetetracarboxylic dianhydride (PTCDA) on $\operatorname{Ag}(110)$ and on $\operatorname{Ag}(111)$ surfaces,${ }^{20}$ the interfacial bonding and charge transfer go along with a hybridization of molecular states, in particular the LUMO, with substrate $s p$ states. This hybridization can be experimentally detected in the momentum distribution curves in the ARPES data. The momentum dependent patterns are immediately connected to the respective molecular wave functions and can-under certain preconditions-be interpreted as a simple Fourier transform of the molecular orbital. ${ }^{37}$ For the chemisorbed PTCDA molecules additional intensity can be observed in the normal emission direction, which is not explained by calculations for the LUMO of the isolated molecule and which can be attributed to a metal $s p$ contribution due to the hybridization between the molecule and metal. ${ }^{20,39}$ The situation for NTCDA/Ag(110) is

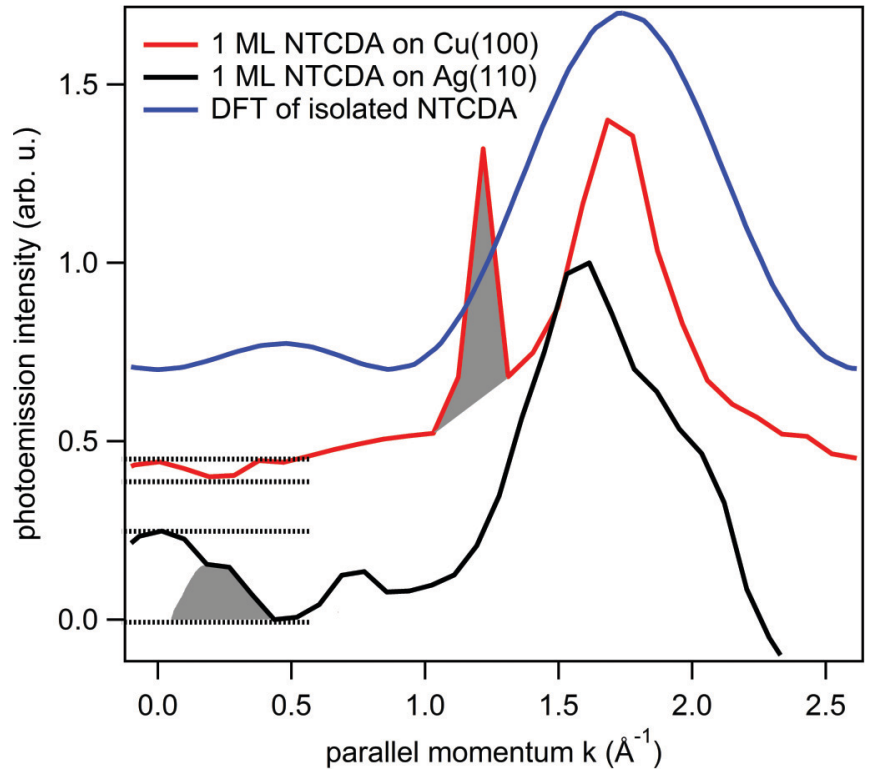

FIG. 4. (Color online) ARPES momentum distribution curves for the LUMO of NTCDA/Ag(110) with $h v=36 \mathrm{eV}$ at $T=100 \mathrm{~K}$ (black line), compared to the LUMO of NTCDA/Cu(100) recorded at $T=60 \mathrm{~K}$ using $\mathrm{He} \mathrm{II}_{\alpha}$ radiation (red line). The curves have been normalized on the intensity maximum around $1.7 \AA^{-1}$. The blue line represents the respective DFT calculation for an isolated NTCDA molecule. The gray shaded areas indicate photoemission signals from $\mathrm{Cu} / \mathrm{Ag} s p$ bands.

very similar. ${ }^{15,21}$ This is illustrated by Fig. 4, which captures the ARPES intensity distribution of the NTCDA LUMO on $\mathrm{Ag}(110)$ (black line) recorded at a binding energy of $0.50 \mathrm{eV}$ along the $k_{1 \overline{1} 0}$ direction, i.e., from $\Gamma$ toward the intensity maximum of the LUMO (see Fig. 5). ${ }^{55}$ Compared to the calculation of the isolated molecule shown in blue, two distinct deviations can be detected. The first one regards the width of the maximum around $k=1.6 \AA^{-1}$, which is significantly smaller for both experimental curves. This is owed to the fact that the momentum distribution curves were determined by integrating over a binding-energy interval of $50 \mathrm{meV}$, which is smaller than the intermolecular band dispersion (discussed in detail in Sec. IIIC). The second deviation of the experimental curves from the calculation for the isolated molecule regards the ARPES intensity at $k_{x}=k_{y}=0$, which does not occur in the DFT calculation. Note that this experimental finding is not influenced by the chosen energy window for integrating the data. As demonstrated in literature for the similar system PTCDA/Ag $(110)^{20}$ this intensity can be taken as a signature for the hybridization of the NTCDA LUMO with substrate $s p$ states. Interestingly, the LUMO in the case of NTCDA/Cu(100) also shows ARPES intensity in normal emission (red line in Fig. 4). However, a comparison of the respective data shows that the substrate contribution is significantly smaller than in the case of NTCDA/Ag(110). Note that this contribution is not visible in the $k_{\|}$-dependent intensity distribution in Fig. 3, since the incident light is purely $p$ polarized in this case while it is a mixture of $s$ and $p$ in the case of Fig. 4. 


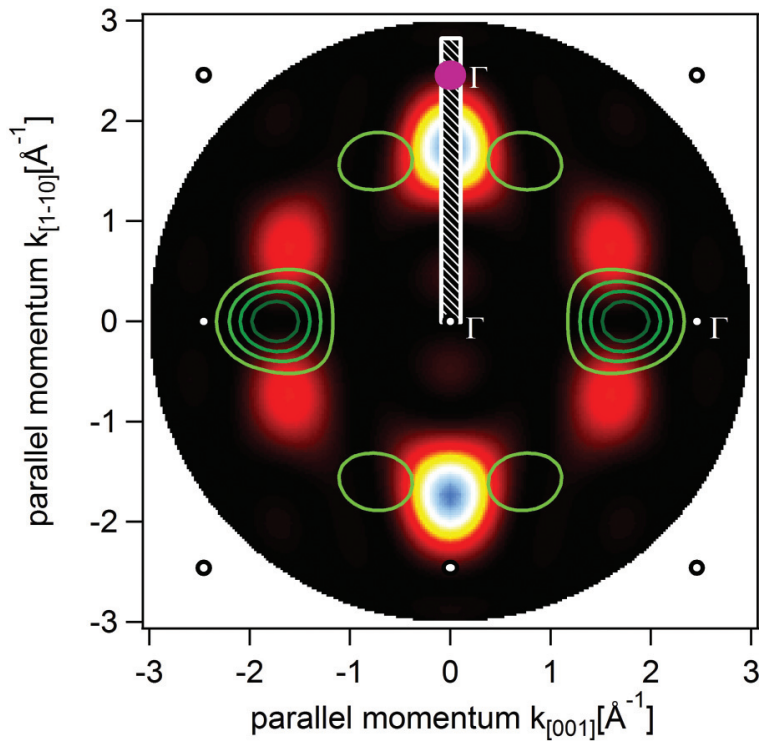

FIG. 5. (Color online) Angular photoemission intensity distribution calculated for the LUMO of an isolated NTCDA molecule. For the monolayer on $\mathrm{Cu}(100)$, two domains with an angle of $90^{\circ}$ exist. Next to the emission from one domain shown in the color code, the photoemission $k_{\|}$distribution of the second domain is shown as a contour plot. Its emission features are therefore also rotated by $90^{\circ}$ against the features of the first domain. For measurements along the [001] direction, only one domain contributes at $k_{[001]} \neq 0 \AA^{-1}$.

A quantitative measure of the relative admixtures can be error prone, since the intensity of the hybridization states is dependent on the orientation of the polarization vector to the molecular coordinates. ${ }^{20,27,39}$ For one domain of the NTCDA/Cu(100), the orientation is identical with NTCDA/Ag(110); for the other domain, the relevant in-plane polarization component is orthogonal. Since the normalization of Fig. 4 is performed on the LUMO maximum around $k_{y}=1.7 \AA^{-1}$, an upper limit of this geometrical factor can be derived from the case, that both domains contribute equally to the hybridization state at the center. But even when taking into account this factor of 2 for NTCDA/Cu(100), the relative strength of the normal emission intensities observed for $\mathrm{Ag}(110)$ and $\mathrm{Cu}(100)$ remains unchanged. This stronger admixture of substrate states to the LUMO in the case of NTCDA/Ag(110) corroborates the findings of a reduced hole lifetime and thus stronger coupling between molecular orbital and substrate electrons in this system with respect to $\mathrm{NTCDA} / \mathrm{Cu}(100)$, which we derived from the line-shape analysis above.

\section{LUMO dispersion}

Figure 6 displays the LUMO regime of the ARPES data of NTCDA/Ag(110) [Fig. 6(a)] and NTCDA/Cu(100) [Fig. 6(b)] plotted against parallel momentum along the $k_{1 \overline{1} 0}$ and $k_{001}$ direction, respectively, thus probing the dispersion along the short molecular axes. To overcome the strong angular intensity variation of $\pi$-conjugated molecules, ${ }^{20,39}$ we rescaled the photoemission intensities for each measured polar angle separately by normalizing it to the average intensity within the LUMO binding-energy region. ${ }^{21}$ In both systems a substantial
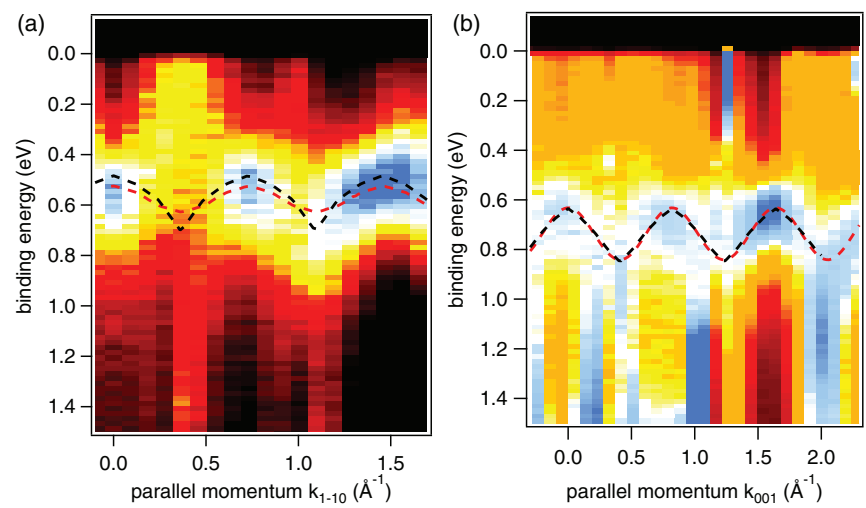

FIG. 6. (Color online) Intensity normalized ARPES data for one monolayer NTCDA (a) on $\operatorname{Ag}(110)$ with $h v=36 \mathrm{eV}$ at $T=100 \mathrm{~K}$

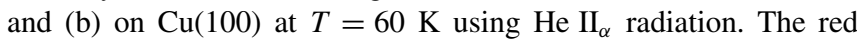
dotted lines show the band dispersion derived theoretically for the LUMO of a free-standing layer of NTCDA in the respective lateral geometries. The black dotted lines show the corresponding LUMO band dispersions for the NTCDA monolayer adsorbed on a $\operatorname{Ag}(110)$ and $\mathrm{Cu}(100)$ slab, respectively. See text for details.

dispersion can be observed. The experimental bandwidth is $180 \mathrm{meV}$ for the LUMO on $\operatorname{Ag}(110)$ and it amounts to a comparable $200 \pm 50 \mathrm{meV}$ on $\mathrm{Cu}(100)$. As we have demonstrated in a previous publication, ${ }^{21}$ the calculated bandwidth of a free-standing NTCDA layer, using the geometry of the NTCDA/ $\operatorname{Ag}(110)$ monolayer, is significantly smaller than in experiment $[100 \mathrm{meV}$, red dotted line in Fig. 6(a)]. Experiment and theory only match if the NTCDA layer is adsorbed on a $\mathrm{Ag}(110)$ slab [black dotted line in Fig. 6(a)], resulting in an enhanced bandwidth of $220 \mathrm{meV}$. Thus, the hybridization of the NTCDA LUMO with Ag states leads to an increased lateral delocalization and thus bandwidth of the resulting hybrid state. ${ }^{56}$ Interestingly, a different scenario occurs if we analyze the NTCDA/ $\mathrm{Cu}(100)$ data along the same approach. Theory predicts the same bandwidth of $210 \mathrm{meV}$ for the more densely packed free-standing [red dotted line in Fig. 6(b)] as for the adsorbed layer [black dotted line in Fig. 6(b)]. In either case the theoretical value coincides with the experiment very nicely, thus indicating that the substrate influences the lateral band dispersion of the LUMO only weakly.

These observations are further corroborated by two aspects. First, the lateral band dispersion due to the direct intermolecular overlap of the LUMO wave functions is clearly expected to be larger in the case of NTCDA/Cu(100) due to the denser packing. Second, the substrate-enhanced lateral dispersion of the LUMO can be assumed to be more pronounced for NTCDA/Ag(110) due to a stronger hybridization with the substrate as evidenced by the decreased lifetime. While no experimental data exist for the vertical bonding distances in the two adsorption systems, DFT calculations can provide some valuable information. A benchmark for density-functional theory calculations with empirical van der Waals correction is given in Ref. 57, estimating the errors for the calculated vertical bonding distances in a similar molecule-metal system to about $5 \%$. In our case the most relevant bonding distance is between the oxygen atoms and the topmost metal atoms. This is $2.32 \AA$ for NTCDA/Ag(110) compared to $2.10 \AA$ for 
NTCDA/Cu(100) (see also Refs. 58 and 59). If the different sizes of the $\mathrm{Ag}$ and $\mathrm{Cu}$ atoms are considered, as expressed in their respective van der Waals radii of $r_{\mathrm{vdw}-\mathrm{Ag}}=1.72 \AA$ and $r_{\mathrm{vdw}-\mathrm{Cu}}=1.40 \AA,{ }^{60,61}$ the so-corrected bonding distance is in fact $0.10 \AA$ shorter for NTCDA/Ag(110) than for NTCDA/Cu(100). Although this difference is relatively small, this may still be taken as an indication for a reduced overlap of the NTCDA LUMO with metal states in the case of adsorption on $\mathrm{Cu}(100)$. Moreover, it must be noted that the more open (110) surface as compared to the (100) may also play a role in the molecule's tendency to hybridize with substrate states.

\section{CONCLUSION}

In conclusion, the comparison of the two highly ordered monolayer systems NTCDA/Ag(110) and NTCDA/Cu(100) provides interesting insight into the complex influences of intermolecular and interfacial bonding on the electronic structure at interfaces. In both cases, the strong interfacial bonding leads to a charge transfer from the metal to the molecule. The occupied LUMO, however, shows characteristic differences between the two substrates. The stronger coupling on $\operatorname{Ag}(110)$ leads to a shorter photohole lifetime, manifested in the larger Lorentzian contribution to the LUMO linewidth in ARPES. The vertical bonding strength is also reflected in the modification of the angular intensity patterns of the LUMO, where a stronger hybridization is observed for NTCDA/Ag(110). The latter can be attributed to a stronger wave-function overlap between the molecule and substrate due to the shorter vertical bonding distance on $\operatorname{Ag}(110)$. Interestingly, the lateral band dispersion of the LUMO is very similar for NTCDA/Ag(110) and for NTCDA/Cu(100), although the prominent mechanisms are different in both cases. While for NTCDA/Ag(110) the dispersion is substantially mediated by the substrate, for NTCDA/Cu(100) the dispersion occurs mainly due to the direct intermolecular wave-function overlap, which is larger due to the denser packing.

\section{ACKNOWLEDGMENTS}

We thank J. Ziroff for providing part of the ARPES data on NTCDA/Ag(110) and M. Arita and K. Shimada (HiSOR) for support during beamtime. We thank D. Hauschild, J. Alsmeier, and H. Harutyunyan for support during beamtime at Elettra. This work was financially supported by the Deutsche Forschungsgemeinschaft (Grants No. GRK 1221 and No. RE1469/9-1) and the Bundesministerium für Bildung und Forschung (Contracts No. 05K10WW2 and No. 03SF0356B). P. Puschnig acknowledges support from the Austrian Science Fund Project No. P23190-N16.
*Corresponding author: achim.schoell@physik.uni-wuerzburg.de

${ }^{1}$ G. Horowitz, Adv. Mater. 10, 365 (1998).

${ }^{2}$ H. Klauk, U. Zschieschang, J. Pflaum, and M. Halik, Nature (London) 445, 745 (2007).

${ }^{3}$ S. Reineke, F. Lindner, G. Schwartz, N. Seidler, K. Walzer, B. Lussem, and K. Leo, Nature (London) 459, 234 (2009).

${ }^{4}$ D. N. Congreve, J. Lee, N. J. Thompson, E. Hontz, S. R. Yost, P. D. Reusswig, M. E. Bahlke, S. Reineke, T. Van Voorhis, and M. A. Baldo, Science 340, 334 (2013).

${ }^{5}$ Y. Zou, L. Kilian, A. Scholl, T. Schmidt, R. Fink, and E. Umbach, Surf. Sci. 600, 1240 (2006).

${ }^{6}$ H. Ishii, K. Sugiyama, E. Ito, and K. Seki, Adv. Mater. 11, 605 (1999).

${ }^{7}$ F. S. Tautz, Prog. Surf. Sci. 82, 479 (2007).

${ }^{8}$ H. Vázquez, W. Gao, F. Flores, and A. Kahn, Phys. Rev. B 71, 041306 (2005).

${ }^{9}$ H. Huang, S. L. Wong, W. Chen, and A. T. S. Wee, J. Phys. D: Appl. Phys. 44, 464005 (2011).

${ }^{10}$ E. Kawabe, H. Yamane, R. Sumii, K. Koizumi, Y. Ouchi, K. Seki, and K. Kanai, Org. Electron. 9, 783 (2008).

${ }^{11}$ N. Koch, Phys. Status Solidi RRL 6, 277 (2012).

${ }^{12} \mathrm{~S}$. Duhm, G. Heimel, I. Salzmann, H. Glowatzki, R. L. Johnson, A. Vollmer, J. P. Rabe, and N. Koch, Nat. Mater. 7, 326 (2008).

${ }^{13}$ S. Braun, W. R. Salaneck, and M. Fahlman, Adv. Mater. 21, 1450 (2009).

${ }^{14}$ J. Hwang, A. Wan, and A. Kahn, Mater. Sci. Eng. R 64, 1 (2009).

${ }^{15}$ A. Bendounan, F. Forster, A. Schöll, D. Batchelor, J. Ziroff, E. Umbach, and F. Reinert, Surf. Sci. 601, 4013 (2007).

${ }^{16}$ H. Yamane, D. Yoshimura, E. Kawabe, R. Sumii, K. Kanai, Y. Ouchi, N. Ueno, and K. Seki, Phys. Rev. B 76, 165436 (2007).
${ }^{17}$ S. Duhm, A. Gerlach, I. Salzmann, B. Bröker, R. Johnson, F. Schreiber, and N. Koch, Org. Electron. 9, 111 (2008).

${ }^{18}$ S. Berkebile, T. Ules, P. Puschnig, L. Romaner, G. Koller, A. J. Fleming, K. Emtsev, T. Seyller, C. Ambrosch-Draxl, F. P. Netzer, and M. G. Ramsey, Phys. Chem. Chem. Phys. 13, 3604 (2011).

${ }^{19}$ B. Stadtmüller, I. Kröger, F. Reinert, and C. Kumpf, Phys. Rev. B 83, 085416 (2011).

${ }^{20}$ J. Ziroff, F. Forster, A. Schöll, P. Puschnig, and F. Reinert, Phys. Rev. Lett. 104, 233004 (2010).

${ }^{21}$ M. Wießner, J. Ziroff, F. Forster, M. Arita, K. Shimada, P. Puschnig, A. Schöll, and F. Reinert, Nat. Commun. 4, 1514 (2013).

${ }^{22}$ A. Schmidt, T. J. Schuerlein, G. E. Collins, and N. R. Armstrong, J. Phys. Chem. 99, 11770 (1995).

${ }^{23}$ G. Nicolay, F. Reinert, S. Schmidt, D. Ehm, P. Steiner, and S. Hüfner, Phys. Rev. B 62, 1631 (2000).

${ }^{24}$ C. Wiemann, M. Patt, I. P. Krug, N. B. Weber, M. Escher, M. Merkel, and C. M. Schneider, Surf. Sci. Nanotechnol. 9, 395 (2011).

${ }^{25}$ M. Escher, N. Weber, M. Merkel, C. Ziethen, P. Bernhard, G. Schönhense, S. Schmidt, F. Forster, F. Reinert, B. Krömker, and D. Funnemann, J. Phys.: Condens. Matter 17, S1329 (2005).

${ }^{26}$ M. Escher, N. Weber, M. Merkel, B. Krömker, D. Funnemann, S. Schmidt, F. Reinert, F. Forster, S. Hüfner, P. Bernhard, C. Ziethen, H. Elmers, and G. Schönhense, J. Electron. Spectrosc. 144-147, 1179 (2005).

${ }^{27}$ M. Wießner, D. Hauschild, A. Schöll, F. Reinert, V. Feyer, K. Winkler, and B. Krömker, Phys. Rev. B 86, 045417 (2012).

${ }^{28}$ R. Fink, D. Gador, U. Stahl, Y. Zou, and E. Umbach, Phys. Rev. B 60, 2818 (1999).

${ }^{29}$ G. Kresse and J. Hafner, Phys. Rev. B 47, 558 (1993).

${ }^{30}$ G. Kresse and D. Joubert, Phys. Rev. B 59, 1758 (1999). 
${ }^{31}$ J. P. Perdew, K. Burke, and M. Ernzerhof, Phys. Rev. Lett. 77, 3865 (1996).

${ }^{32}$ S. Grimme, J. Comput. Chem. 27, 1787 (2006).

${ }^{33}$ P. E. Blöchl, Phys. Rev. B 50, 17953 (1994).

${ }^{34}$ H. J. Monkhorst and J. D. Pack, Phys. Rev. B 13, 5188 (1976).

${ }^{35}$ M. Methfessel and A. T. Paxton, Phys. Rev. B 40, 3616 (1989).

${ }^{36}$ J. Neugebauer and M. Scheffler, Phys. Rev. B 46, 16067 (1992).

${ }^{37}$ P. Puschnig, S. Berkebile, A. J. Fleming, G. Koller, K. Emtsev, T. Seyller, J. D. Riley, C. Ambrosch-Draxl, F. P. Netzer, and M. G. Ramsey, Science 326, 702 (2009).

${ }^{38}$ C. Stadler, S. Hansen, A. Schöll, T.-L. Lee, J. Zegenhagen, C. Kumpf, and E. Umbach, New J. Phys. 9, 50 (2007).

${ }^{39}$ P. Puschnig, E.-M. Reinisch, T. Ules, G. Koller, S. Soubatch, M. Ostler, L. Romaner, F. S. Tautz, C. Ambrosch-Draxl, and M. G. Ramsey, Phys. Rev. B 84, 235427 (2011).

${ }^{40}$ S. Kera, S. Tanaka, H. Yamane, D. Yoshimura, K. Okudaira, K. Seki, and N. Ueno, Chem. Phys. 325, 113 (2006).

${ }^{41}$ M. Dauth, T. Körzdörfer, S. Kümmel, J. Ziroff, M. Wiessner, A. Schöll, F. Reinert, M. Arita, and K. Shimada, Phys. Rev. Lett. 107, 193002 (2011).

${ }^{42}$ J. Sauther, J. Wüsten, S. Lach, and C. Ziegler, J. Chem. Phys. 131, 034711 (2009).

${ }^{43}$ N. Ueno and S. Kera, Prog. Surf. Sci. 83, 490 (2008).

${ }^{44}$ J.-L. Brédas, D. Beljonne, V. Coropceanu, and J. Cornil, Chem. Rev. 104, 4971 (2004).

${ }^{45}$ S. Kera, H. Yamane, and N. Ueno, Prog. Surf. Sci. 84, 135 (2009).

${ }^{46}$ S. Duhm, Q. Xin, S. Hosoumi, H. Fukagawa, K. Sato, N. Ueno, and S. Kera, Adv. Mater. 24, 901 (2012).

${ }^{47}$ S. Kera, S. Hosoumi, K. Sato, H. Fukagawa, S.-i. Nagamatsu, Y. Sakamoto, T. Suzuki, H. Huang, W. Chen, A. T. S. Wee, V. Coropceanu, and N. Ueno, J. Phys. Chem. C (2013), doi: 10.1021/jp4032089.
${ }^{48}$ S. Kera, H. Yamane, I. Sakuragi, K. K. Okudaira, and N. Ueno, Chem. Phys. Lett. 364, 93 (2002).

${ }^{49}$ H. Yamane, S. Nagamatsu, H. Fukagawa, S. Kera, R. Friedlein, K. K. Okudaira, and N. Ueno, Phys. Rev. B 72, 153412 (2005).

${ }^{50}$ J. K. Norsko, Rep. Prog. Phys. 53, 1253 (1990).

${ }^{51}$ V. Coropceanu, M. Malagoli, D. A. da Silva Filho, N. E. Gruhn, T. G. Bill, and J. L. Brédas, Phys. Rev. Lett. 89, 275503 (2002).

${ }^{52}$ P. B. Paramonov, V. Coropceanu, and J.-L. Brédas, Phys. Rev. B 78, 041403 (2008).

${ }^{53}$ R. Matzdorf, Appl. Phys. A 63, 549 (1996).

${ }^{54}$ A. Eiguren, B. Hellsing, F. Reinert, G. Nicolay, E. V. Chulkov, V. M. Silkin, S. Hüfner, and P. M. Echenique, Phys. Rev. Lett. 88, 066805 (2002).

${ }^{55}$ Note that ARPES measurements, especially of the LUMO, are still comparable to the measurements on $\operatorname{Ag}(110)$, since the angular emission pattern of the LUMO vanishes for one of the two symmetry equivalent domains (Fig. 5).

${ }^{56}$ Note that the values of 60 and $120 \mathrm{meV}$ for the band width of the free-standing and adsorbed NTCDA layer on $\mathrm{Ag}(110)$, respectively, given in Ref. 21 were calculated for a geometry which was not in the global energy minimum. In the refined geometry utilized here, the free-standing layer has a bandwidth of $100 \mathrm{meV}$ and the adsorbed layer has a bandwidth of $220 \mathrm{meV}$.

${ }^{57}$ O. Bauer, G. Mercurio, M. Willenbockel, W. Reckien, C. Heinrich Schmitz, B. Fiedler, S. Soubatch, T. Bredow, F. S. Tautz, and M. Sokolowski, Phys. Rev. B 86, 235431 (2012).

${ }^{58}$ A. Alkauskas, A. Baratoff, and C. Bruder, Phys. Rev. B 73, 165408 (2006).

${ }^{59}$ A. Abassi, Ph.D. thesis, Chemnitz University of Technology, 2010.

${ }^{60}$ A. Bondi, J. Phys. Chem. 68, 441 (1964).

${ }^{61}$ I. Kröger, B. Stadtmüller, C. Kleimann, P. Rajput, and C. Kumpf, Phys. Rev. B 83, 195414 (2011). 